\title{
BECA DE NIVELACIÓN ACADÉMICA: RESULTADOS DE UN PROGRAMA DE INTERVENCIÓN EN ESTUDIANTES DE ORIGEN VULNERABLE EN LA UNIVERSIDAD DE CONCEPCIÓN
}

\author{
Valentina Soto Hernández \\ Universidad Autónoma de Barcelona, Barcelona - España \\ Claudio Díaz Larenas** \\ Universidad de Concepción, Concepción - Chile \\ María Teresa Chiang Salgado *** \\ Universidad de Concepción, Concepción - Chile
}

RESUMEN: El artículo presenta los resultados de la evaluación de logros académicos de 120 beneficiarios del programa Beca de Nivelación Académica implementado por la Universidad de Concepción. El propósito del programa es apoyar la retención y mejorar el rendimiento académico de estudiantes universitarios de primer año de origen socioeconómico vulnerable. Se utilizó una metodología cuantitativa y se definió un estudio correlacional, cuyos resultados indican que la retención alcanzó un 87\%, mientras que el promedio de notas fue un 5.1, mejorando los resultados anteriores y logrando los indicadores comprometidos. La prueba de hábitos de estudios demostró constituir el mejor predictor del rendimiento académico. Estos resultados fueron complementados con dos focus group, que revelaron la alta valoración de los estudiantes hacia el programa como una instancia facilitadora de su adaptación a las exigencias universitarias. Finalmente, se reflexiona respecto a las políticas públicas que buscan fortalecer la equidad en la educación superior en Chile.

Palabras clave: Estudiantes universitarios. Equidad. Rendimiento académico. Retención.

\footnotetext{
"Doctorante en Sociología, Universidad Autónoma de Barcelona.

E-mail:<sotohernandez.valentina@gmail.com>.

"Doctor en Educación, Universidad de Concepción, Chile. Profesor Investigador y Jefe de la Unidad de Investigación y Desarrollo Docente, Dirección de Docencia de la Universidad de Concepción.

E-mail:<claudiodiaz@udec.cl>.

"**Magister en Ciencias. Directora del Centro de Apoyo al Desarrollo del Estudiante. Subdirectora de Dirección de Docencia, Universidad de Concepción.

E-mail:<mchiang@udec.cl>.
} 


\section{BOLSA DE NIVELAMENTO ACADÊMICO: RESULTADOS DE UM PROGRAMA DE INTERVENC̣̃̃O EM ESTUDANTES DE ORIGEM VULNERÁVEL NA UNIVERSIDADE DE CONCEPCIÓN}

RESUMO: $\mathrm{O}$ artigo apresenta os resultados da avaliação do desempenho acadêmico de 120 beneficiários do programa de Bolsa de Nivelação Acadêmica implementado pela Universidade de Concepción. O objetivo do programa é apoiar a permanência e melhorar o rendimento acadêmico de estudantes universitários do primeiro ano de origem socioeconômica vulnerável. Utilizou-se uma metodologia quantitativa e definiu-se um estudo correlacional, cujos resultados indicam que a permanência alcançou $87 \%$, enquanto a média de notas foi de 5,1, melhorando os resultados anteriores e atingindo os indicadores previstos. A prova de hábitos de estudos demonstrou ser o melhor preditor do rendimento acadêmico. Esses resultados foram complementados com dois grupos focais, os quais revelaram a grande valorização do programa pelos estudantes como uma instância facilitadora de sua adaptação às exigências universitárias. Finalmente, reflete-se sobre as políticas públicas que buscam fortalecer a equidade na educação superior no Chile.

Palavras-chave: Estudantes universitários. Equidade. Rendimento acadêmico. Permanência.

\section{ACADEMIC LEVELING PROGRAM: FINDINGS OF AN INTERVENTION PROGRAM ON SOCIALLY VULNERABLE STUDENTS AT UNIVERSIDAD DE CONCEPCIÓN}

ABSTRACT: This paper presents the findings of the assessment of 120 students' academic performance, who benefited from an academic leveling program implemented by the University of Concepción. The purpose of this program is to support the stay and to improve the academic performance of first year university students of socioeconomic vulnerable origin. A quantitative methodology was used and a correlational study was defined; the results show the stay reached $87 \%$, while participants' averaged grade was 5.1 on a scale from 1 to 7 . This allows improving participants' past results and accomplishing the expected indicators. The study habits test proved to be the best predictor of academic achievement. These results were complemented by two focus groups that show the students' high value of the program as a facilitating instance of their adaptation to the university demands. Finally, there is a reflection on public policies that seek to strengthen higher education equity in Chile.

Keywords: University students. Equity. Academic achievemnet. Retention. 


\section{INTRODUCCIÓN}

Durante los últimos treinta y cinco años, y luego de una reforma legal impulsada por la dictadura militar (1973-1990), el sistema de educación universitario chileno ha experimentado transformaciones radicales. Una de las más relevantes es el crecimiento explosivo de la matrícula, que ha pasado desde hasta 118978 estudiantes en 1980 a 1215413 en 2014 (SIES, 2014; BERNASCONI; ROJAS, 2004). Este crecimiento ha implicado una transformación en el perfil de los estudiantes, quienes han sido llamados estudiantes no tradicionales, estudiantes de primera generación o estudiantes de origen vulnerable, y pertenecen a grupos sociales tradicionalmente marginados de la educación superior.

Los estudiantes de origen vulnerable enfrentan diversas limitaciones para acceder a la educación superior. En efecto, los logros escolares se encuentran fuertemente asociados al origen socioeconómico. Para ingresar a la universidad, los estudiantes deben rendir la Prueba de Selección Universitaria (PSU), cuyos resultados se encuentran determinados por factores como la escolaridad de los padres y el ingreso familiar (CONTRERAS; CORBALÁN; REDONDO et al., 2007; MANZI, 2006).

Los cambios introducidos en la PSU que buscaban igualar las condiciones entre estudiantes de distinto origen social, no han conseguido los efectos esperados. De acuerdo a Koljatic y Silva (2010, p. 130), "el aumento de contenidos en las pruebas PSU no ha favorecido a los alumnos de sectores más vulnerables, sino que, por el contrario, los ha perjudicado". De igual forma, la modalidad educacional Técnico-Profesional ${ }^{1}$ ha sido fuertemente perjudicada (PEARSON, 2013; KOLJATIC; SILVA 2010).

Otra limitación en el acceso de los grupos de menores ingresos es el alto costo de la educación superior en Chile. La comparación de los aranceles con el ingreso per cápita demuestra que "el costo de los estudios es considerablemente más alto que en los países de la OCDE que cobran aranceles" (OCDE, 2009, p. 121).

Una vez en la universidad, los datos indican que los estudiantes de sectores de bajos ingresos y provenientes de escuelas municipales tienen mayores probabilidades de desertar del sistema, y los que logran graduarse demoran más tiempo en finalizar (OCDE, 2009). En consecuencia, y a pesar de la explosión en la matrícula en educación superior, persiste la falta de equidad en el acceso a la educación superior (ESPINOZA; GONZÁLEZ; LATORRE, 2009). 
Los programas de compensación o de nivelación han surgido de manera incipiente durante los últimos años, impulsados desde las universidades y el Ministerio de Educación (en adelante, MINEDUC). Su propósito es mejorar la retención y el rendimiento académico, especialmente de estudiantes más vulnerables. En este artículo se presenta una investigación acerca de un grupo de beneficiarios de la Beca de Nivelación Académica en una universidad chilena, que busca profundizar en sus características sociodemográficas y analizar su rendimiento académico durante el primer año en la universidad.

El artículo se divide en cuatro partes. Primero, en el marco de referencia, se analizan algunos rasgos del sistema de educación chileno, para brindar un contexto que permita comprender el surgimiento del Programa Beca de Nivelación Académica. A continuación, se detalla la metodología de investigación. En la tercera parte, se analizan los resultados del programa, a través de una caracterización sociodemográfica, rendimiento académico y percepción de la participación en el programa. Finalmente, se discuten los resultados y se realizan sugerencias a modo de conclusión.

\section{EXPANSIÓN E INEQUIDAD EN LA EDUCACIÓN SUPERIOR CHILENA}

El proceso de expansión de la educación superior ha implicado un notable incremento de cobertura para la población de 18 a 24 años. En 1980, la matrícula total ascendía a 118 978, que corresponde a una cobertura de 7.5\%. En el año 1990, la cobertura prácticamente se duplicó, llegando a 14.2\%. En 2013, la matrícula superó el millón de estudiantes 1112416 - con un 45.8\% de cobertura (ESPINOZA, 2015).

El estudio de Zapata, Tejeda y Rojas (2011) analizó la distribución social de la matrícula en educación superior durante los últimos cinco años, observando un crecimiento en la participación de los quintiles I y II, en una tendencia que se consolida. No obstante, el tipo de enseñanza superior "escogida" no es el mismo según el origen familiar. Los jóvenes de sectores más favorecidos, de quintiles IV y $\mathrm{V}$, estudian generalmente en la universidad; mientras que los jóvenes de sectores vulnerables estudian mayoritariamente en Centros de Formación Técnica (PNUD, 2005). El acceso de estudiantes de los dos primeros quintiles se explicó por el acceso a instituciones técnicas $^{2}$ (DONOSO-DÍAZ; CÁNCINO, 2007; PNUD, 2005).

La Encuesta de Caracterización Socioeconómica CASEN (CHILE, 2016) señala que la cobertura de la Educación Superior es de $27.4 \%$ para el quintil I, mientras que en el quintil $\mathrm{V}$ es un $57.5 \%$. Los datos presentados dan cuenta de un incremento gradual 
y sostenido en el tiempo del acceso a grupos que anteriormente no ingresaban a este nivel educativo. A pesar de estos avances, se produce un fenómeno de segmentación social de los estudiantes de acuerdo a su origen familiar (ESPINOZA; GONZÁLEZ; LATORRE, 2009).

\section{SEGMENTACIÓN DEL SISTEMA DE EDUCACIÓN SUPERIOR}

De acuerdo a Brunner (2009), el sistema de educación superior se organiza a partir de la competencia por estudiantes, lo que implica dos fenómenos cuyo eje principal es la segmentación del sistema. El primer fenómeno es la expansión segmentada, producida por estrategias de expansión institucional, como la creación sedes y programas de estudio.

El segundo fenómeno es la estratificación escalonada. El crecimiento de la matrícula se desarrolla en forma de cascada, en la cual las universidades son ubicadas en un orden decreciente de selectividad. En la parte superior, se encuentran las universidades con una fuerte selección académica, que aceptan estudiantes con los mejores puntajes en la PSU. En cambio, los estudiantes con menores resultados se inscriben en universidades menos selectivas, así sucesivamente, hasta llegar a universidades que no seleccionan a sus alumnos. ${ }^{3}$ El efecto cascada

constituye al mismo tiempo un proceso de selección social como muestra la distribución socioeconómica de los puntajes PSU, la cual a su turno expresa las diferentes trayectorias escolares de los jóvenes determinadas por su origen familiar (BRUNNER, 2009, p. 386).

En consecuencia, y a pesar de la sostenida incorporación de estudiantes de los quintiles de más bajos ingresos, la selectividad de las instituciones se constituye en una estratificación no sólo académica, sino también en "un dispositivo de estratificación socioeconómica de los alumnos” (BRUNNER, 2009, p. 387).

\section{LAS BARRERAS AL INTERIOR DE LA EDUCACIÓN SUPERIOR}

Una vez analizadas las barreras de ingreso al sistema de educación superior y la composición social derivada de su estructura, es importante señalar que las investigaciones muestran que los estudiantes experimentan desigualdades educativas durante su formación universitaria.

Un fenómeno relevante se produce en torno a la deserción universitaria. El estudio cualitativo de Canales y De Los Ríos (2007), 
realizado en Chile, indica que este fenómeno se vincula al origen social de los estudiantes, tanto en la deserción temporal como permanente. En la primera, se identifican dificultades de adaptación al ritmo universitario y problemas de rendimiento académico, experimentado especialmente por estudiantes provenientes de establecimientos particulares subvencionados y municipales. Otro aspecto importante se refiere al desconocimiento acerca de las oportunidades laborales de las carreras escogidas. En la deserción permanente, por su parte, se destacan las causas económicas asociadas a problemas familiares, dando cuenta de la vulnerabilidad de los estudiantes. La deserción permanente

no constituye una deserción buscada o deseada por los estudiantes. Al contrario, la decisión de dejar de estudiar es percibida como difícil debido a que existen expectativas familiares y personales (CANALES; DE LOS RÍOS, 2007, p. 191-192).

El Centro de Microdatos de la Universidad de Chile (2008) destaca que las principales causas de deserción son los problemas vocacionales, la situación económica de la familia y el rendimiento académico.

La escolaridad de los padres ha sido identificada como un factor que influye fuertemente en las posibilidades educativas de los hijos; los llamados estudiantes de primera generación, cuyos padres no accedieron a la educación superior, experimentan dificultades para ingresar y luego para permanecer en la universidad (CPCE, 2009; GROLEAU et al., 2010; CHEN; CARROLL, 2005; BEAUD, 2002; WARBURTON et al., 2001). Para el caso chileno, Urzúa (2012) plantea que, del total de jóvenes que no terminaron sus estudios superiores, un $74 \%$ era de primera generación. De este modo, para los hijos de padres universitarios, la probabilidad de obtener un título universitario es de un 70\%; mientras que, para los estudiantes de primera generación, la probabilidad alcanza un 38\%. En un estudio de seguimiento de una cohorte -2003 y 2009-, Castillo y Cabezas (2010) señalan que, del total de jóvenes que desertaron del sistema, un $83 \%$ correspondió a estudiantes de primera generación.

Leyton, Vázquez y Fuenzalida (2012) sostienen que los estudiantes universitarios provenientes de sectores vulnerables reconocen una precaria formación escolar, comparada con estudiantes de estratos altos, lo que se expresa en un "sentimiento de desigualdad". Asimismo, estas deficiencias son percibidas como un obstáculo para alcanzar el rendimiento académico esperado. De acuerdo a Canales y De Los Ríos (2009), los estudiantes identifican deficiencias en aspectos tales como dominio de contenidos, hábitos de estudio, manejo de idiomas, redacción y cultura general. 


\section{POLÍTICAS PÚBLICAS PARA MEJORAR LA RETENCIÓN DE ESTUDIANTES VULNERABLES}

Hasta muy recientemente, de acuerdo a Donoso-Díaz, Donoso y Arias (2010), la deserción y la retención de los estudiantes no estaban consideradas como una preocupación para las instituciones de educación superior, y, en consecuencia, el fracaso académico era entendido como un asunto individual de los estudiantes. "El fracaso en la enseñanza superior se privatiza, (...) porque se asigna la responsabilidad casi absoluta al estudiante" (DONOSO-DÍAZ; DONOSO; ARIAS, 2010, p. 20). De acuerdo a Canales y De Los Ríos (2009), las iniciativas de apoyo identificadas por su estudio, destinadas a disminuir la deserción, eran puntuales y desarrolladas a escala limitada. Para Donoso-Díaz, Donoso y Arias (2010), en la medida que no existan iniciativas compensadoras, el fracaso será más significativo para los estudiantes de menor capital económico y cultural, contribuyendo así a la reproducción de desigualdades sociales.

Las políticas públicas destinadas a mejorar la equidad en educación superior fueron orientadas al establecimiento de un sistema de financiamiento, asociado a programas de ayuda estudiantil, como créditos y becas ( ESPINOZA; GONZÁLEZ; LATORRE, 2009). Sin embargo, como se indicó, las causas económicas no son las únicas variables que inciden en el abandono del sistema de educación superior. Las instituciones han comenzado a atender de manera incipiente los problemas académicos y de integración que afectan a los estudiantes más vulnerables. En este sentido, Letelier et al. (2010, p. 93) plantean:

Las acciones institucionales asociadas a la equidad se expresan en la existencia de programas institucionales orientados a satisfacer necesidades específicas de algunos alumnos y alumnas, y que han nacido generalmente en forma reactiva. Dichas acciones no obedecen a políticas institucionales explícitas y amplias comparables al $[\ldots]$ aseguramiento de la calidad o vinculación con el medio.

De acuerdo a los autores, los programas institucionales se enfocan principalmente en aspectos económicos y de forma complementaria en la atención académica. Por su parte, Alvarado et al. (2010) identificaron programas que buscan aumentar la retención a través de la inducción a la vida universitaria y de la nivelación de competencias básicas y/o genéricas.

A pesar de estos antecedentes, algunas instituciones pioneras en la preocupación por la inequidad desarrollaron programas propedéuticos, que constituyeron una iniciativa relevante posteriormente replicada por otras instituciones. Estos programas, impulsados a partir de 2007, buscan corregir los problemas de equidad en el acceso a la universidad 
que experimentan los estudiantes de origen socioeconómico vulnerable; por ello sus criterios de selección se basan en la trayectoria escolar, es decir, se selecciona a estudiantes de alto rendimiento académico, sin considerar los resultados de la PSU (ROMÁN, 2013).

\section{EL CASO DE LA BECA DE NIVELACIÓN ACADÉMICA}

Una de las primeras iniciativas dirigidas por el Ministerio de Educación destinada a mejorar la retención fue la Beca de Nivelación Académica (en adelante, BNA). Su propósito es nivelar competencias de estudiantes desfavorecidos que ingresan al primer año de educación superior. El programa, establecido en el año 2012, tiene el objetivo de "apoyar el ingreso y la permanencia en la educación superior de los estudiantes beneficiarios [...], aumentar la retención y favorecer el incremento del nivel de logros y rendimiento académico de estos estudiantes" (CHILE, 2014, p. 2). Este programa se materializa mediante dos procesos. Primero, el Ministerio de Educación realiza una convocatoria pública para que las Instituciones de Educación Superiores presenten sus propuestas, entre ellas se evalúa y selecciona a las mejores. Luego, entre los postulantes a becas y créditos, se designa como beneficiarios de la BNA a estudiantes matriculados en instituciones con programas adjudicados (CHILE, 2014).

El proceso para determinar a los estudiantes beneficiarios es el siguiente: las universidades postulan a los fondos del Programa Beca de Nivelación Académica del Ministerio de Educación. En el caso analizado, la universidad expresó en su proyecto que "la institución puede atender a 120 estudiantes" de determinadas carreras. ${ }^{4}$ Una vez evaluado y adjudicado el proyecto, el MINEDUC selecciona a los beneficiarios en base a tres criterios: egresar de enseñanza media desde establecimientos educacionales municipales y particulares subvencionados; poseer un alto rendimiento escolar; y pertenecer a los tres primeros quintiles de ingreso familiar. ${ }^{5}$ En este proceso, la universidad no participa ni tiene injerencia. El MINEDUC cruza la información disponible de los estudiantes que ingresan a la universidad, selecciona de acuerdo a los criterios mencionados y envía la nómina a la institución, para que se gestione la incorporación de los estudiantes al proyecto.

A diferencia de otros programas destinados a mejorar los indicadores de abandono escolar, como la Beca de Apoyo a la Retención Escolar de Enseñanza Media, el Programa Beca de Nivelación Académica no realiza transferencias de dinero ni provee beneficios materiales. Los estudiantes beneficiarios del programa 
acceden a una red de apoyo académico en la universidad -constituida por profesionales, profesores y estudiantes de cursos superiores- a través de su participación en tutorías, asistencia a charlas y talleres, y actividades de inserción en la vida universitaria. En general, los estudiantes BNA son beneficiados con becas de arancel debido a su situación socioeconómica; no obstante, la obtención de una beca de arancel no implica la obtención de una BNA.

En síntesis, la BNA tiene un doble propósito: mejorar el rendimiento académico (y la permanencia en la universidad) de los estudiantes de origen vulnerable y los indicadores institucionales de retención, particularmente en aquellas carreras con antecedentes de baja tasa de retención.

\section{CÍRCULO-CADE}

La Universidad de Concepción ha impulsado una serie de iniciativas orientadas a apoyar a los estudiantes de origen social desfavorecido para que logren mejores resultados académicos. La primera iniciativa fue la implementación del Centro de Apoyo al Desarrollo del Estudiante (en adelante, CADE), financiado por el MINEDUC y destinado a ofrecer a los estudiantes un apoyo integral. Su primer logro fue la construcción y habilitación de un espacio físico, que incluye oficinas y un auditorio.

El primer proyecto para implementar el programa BNA en la universidad benefició a 87 estudiantes de primer año, durante al año académico 2012. El año siguiente, un nuevo proyecto ofreció apoyo a 120 estudiantes de carreras prioritarias, con el propósito de promover el desarrollo de competencias personales y académicas para mejorar los indicadores de retención en la universidad.

En el año 2014, se implementó el proyecto "Círculo-CADE: programa integral para facilitar la inserción y la permanencia en la universidad". El presente artículo da cuenta de los resultados de este proyecto, es decir, la tercera versión de la BNA en la Universidad de Concepción.

El proyecto se focalizó en las carreras de pedagogía, junto con otras carreras que presentan menos de 550 puntos de corte en la PSU y cuyos estudiantes alcanzan habitualmente un bajo rendimiento en primer año, de acuerdo a los registros de la universidad. Los estudiantes de estas carreras presentaban una alta vulnerabilidad social y académica, lo que configura riesgo de bajo rendimiento. El objetivo general ${ }^{6}$ del programa fue "desarrollar el Programa CírculoCADE UdeC 2014 con 120 estudiantes beneficiarios, para favorecer 
sus competencias personales y académicas a través de un proceso integrado de inserción y acompañamiento". Los objetivos específicos del programa fueron:

a) fortalecer en los estudiantes las habilidades sociales y académicas básicas para disminuir el impacto que genera la transición a la educación superior y así logren una buena inserción;

b) nivelar los contenidos de ciencias básicas y mejorar la comunicación oral y escrita para que puedan lograr rendimientos académicos adecuados.

Elproyectoimplementó una estrategiade apoyo yacompañamiento a los estudiantes, mediante acciones destinadas a fortalecer sus habilidades socioacadémicas y nivelar sus conocimientos de ciencias básicas y comunicación oral y escrita. La estrategia se implementó gracias a la continuación de acciones exitosas de los proyectos anteriormente adjudicados y a la incorporación de iniciativas nuevas.

Algunas de las acciones previamente realizadas fueron:

a) recibir tempranamente a los estudiantes beneficiarios para interiorizarlos de los alcances de la Beca de Nivelación Académica, motivarlos y comprometerlos;

b) establecer una red de apoyo con estudiantes tutores, seleccionados, capacitados y coordinados por CADE, quienes deberán mantener una comunicación fluida tanto con los estudiantes a su cargo, como con los jefes de carrera y equipo CADE;

c) ofrecer apoyo permanente de psicólogos, trabajadores sociales y psicopedagogos, integrados con los servicios de apoyo intrauniversitarios; d) ofrecer de forma permanente las charlas y talleres dictados por el CADE para todos los estudiantes UdeC.

Entre las nuevas acciones se destacan:

a) implementar módulos tutoriales accesibles vía plataforma virtual, con contenidos de matemáticas, física y biología apoyados con estudiantes monitores;

b) dictar el taller "La Oración tópico" (construcción de textos breves) y el curso "Comprensión Textual" para mejorar la producción y la comprensión de textos respectivamente.

Las acciones mencionadas se encuentran asociadas a una red conformada por los profesionales CADE, estudiantes tutores, profesores de asignatura y jefes de carrera. Esta red se encuentra a cargo de realizar el seguimiento del rendimiento académico, que es complementado con la información proporcionada por las plataformas institucionales de administración curricular.

Finalmente, cabe mencionar que los estudiantes tutores son seleccionados en sus carreras y capacitados por los profesionales del 
CADE. Sus principales funciones son: apoyar académicamente a los estudiantes en las asignaturas de ciencias básicas; orientar su inserción en la vida universitaria; motivar su participación en las actividades del programa; y monitorear su rendimiento académico.

\section{INDICADORES DEL CÍRCULO-CADE}

Para lograr los objetivos de la BNA, el MINEDUC propone indicadores generales, consignados en el formulario de postulación. Por su parte, las cifras de cada indicador, tanto las líneas base como las metas, son calculadas por las instituciones a partir de sus registros. En el caso analizado, la postulación se realizó en noviembre de 2013, pero fue necesario utilizar los datos de la cohorte de 2012, puesto que en esa fecha el año académico 2013 no había finalizado y los datos de ese año no estaban disponibles. Por tanto, los valores base indicados en la Tabla 1 corresponden a la cohorte del año 2012.

Las metas establecidas corresponden a una estimación realista de las posibilidades de mejoramiento de los indicadores basada en el comportamiento histórico de las cohortes, considerando variables como puntaje PSU, perfil de los estudiantes y rendimiento académico. El objetivo del programa es cumplir las metas propuestas, aunque estas pueden o no lograrse. En este sentido, cabe destacar que la línea base corresponde a la última cohorte no intervenida por el programa, por lo tanto, no se realiza una comparación entre estudiantes beneficiarios. Entonces, las metas establecidas, aunque aparentemente no sean tan diferentes de la línea base, implican, en efecto, un gran desafío.

En el caso del Círculo-CADE, los indicadores comprometidos se presentan en la siguiente tabla.

TABLA 1 - Indicadores proyecto BNA Universidad de Concepción

\begin{tabular}{|c|c|c|c|}
\hline Indicador & Fórmula de cálculo & $\begin{array}{l}\text { Valor base } \\
\text { (año 2012) }\end{array}$ & $\begin{array}{l}\text { Meta año } \\
2014\end{array}$ \\
\hline $\begin{array}{l}\text { Tasa de retención de estudiantes } \\
\text { beneficiarios del programa BNA. }\end{array}$ & $\begin{array}{l}\mathrm{N}^{0} \text { estudiantes beneficiarios del } \\
\text { programa BNA que continúan estudios } \\
\text { en el tercer semestre / } \mathrm{N}^{0} \text { estudiantes } \\
\text { beneficiarios del programa BNA }\end{array}$ & $85 \%$ & $87 \%$ \\
\hline $\begin{array}{l}\text { Tasa de aprobación de estudiantes } \\
\text { beneficiarios del programa BNA } \\
\text { (estudiantes que aprueban todos } \\
\text { sus créditos) }\end{array}$ & $\begin{array}{l}\mathrm{N}^{0} \text { estudiantes beneficiarios BNA } \\
\text { que aprueban todos sus créditos I } \\
\mathrm{N}^{0} \text { estudiantes beneficiarios BNA }\end{array}$ & $25 \%$ & $30 \%$ \\
\hline $\begin{array}{l}\text { Tasa de rendimiento académico de } \\
\text { estudiantes beneficiarios del programa } \\
\text { BNA (Promedio ponderado de notas). }\end{array}$ & $\begin{array}{l}\text { Promedio ponderado de todos los } \\
\text { alumnos beneficiarios del programa } \\
\text { BNA }\end{array}$ & 4.38 & 4.7 \\
\hline
\end{tabular}


La retención, en términos generales, señala que un estudiante permanece matriculado en la institución. El indicador de retención de primer año corresponde al porcentaje de estudiantes que están matriculados en el tercer semestre de la carrera a la cual ingresaron. Los beneficiarios del programa ingresaron a sus carreras el primer semestre de 2014. Aquellos que estaban matriculados en la misma carrera el tercer semestre de la carrera (abril de 2015) fueron considerados para el cálculo del indicador.

En cuanto al rendimiento académico, las universidades chilenas utilizan la escala de notas de 1.0 a 7.0, donde la nota 4.0 es el límite de la aprobación, y una nota inferior corresponde a reprobación. En el caso de la Universidad de Concepción, el rendimiento académico se relaciona con la aprobación de créditos y asignaturas. Cada asignatura tiene determinado un cierto número de créditos, cuando se aprueba la asignatura, se aprueban esos créditos. Un "buen o adecuado rendimiento académico" significa la aprobación de todas las asignaturas y créditos inscritos, en el tiempo previsto. Un estudiante con "buen rendimiento académico" aprobó todas las asignaturas inscritas. Un estudiante con "muy buen rendimiento", además de aprobar, obtuvo altas notas. Por ejemplo, cuando un estudiante aprueba todas sus asignaturas y finaliza oportunamente la carrera, alcanzando un promedio sobre 5.7, califica como candidato a Premio Universidad de Concepción.

\section{METODOLOGÍA}

La metodología corresponde a un estudio de tipo cuantitativo y correlacional, orientado a examinar los resultados de la intervención realizada por el CADE, en la que participaron 120 beneficiarios de la BNA 2014, estudiantes de la Universidad de Concepción. La población en estudio corresponde a estudiantes de primer año pertenecientes a 22 carreras. ${ }^{7}$ Este programa se focalizó principalmente en carreras de la Facultad de la Educación, que representan el 63.6\% del total, seguidas por carreras de las facultades de Ciencias Naturales y Oceanográficas, y de Ciencias Forestales, con un 13.6\% respectivamente; el resto de las carreras provienen de distintas facultades.

Luego de realizada la intervención, se realizó una evaluación ex post del programa, para la cual se analizaron los resultados académicos de los estudiantes, medidos de acuerdo a los indicadores mencionados. Este análisis constituye el objetivo general de la investigación presentada en el artículo; adicionalmente, se analizaron 
las características sociodemográficas y académicas de los estudiantes y las percepciones de su participación en el Círculo-CADE, como una manera de presentar una perspectiva más completa de la implementación del programa.

Los datos analizados fueron obtenidos de:

a) base de datos del Programa Círculo-CADE;

b) aplicación de dos focus group a los estudiantes beneficiarios de la beca; c) encuesta web anónima a los jóvenes beneficiarios al término del segundo semestre.

Los datos cuantitativos, provenientes de la base de datos y la encuesta web, fueron procesados con el programa SPSS versión 20.0. Se utilizaron estadística descriptiva, para realizar la caracterización sociodemográfica y académica de los estudiantes, y estadística multivariada, para encontrar correlaciones estadísticamente significativas entre rendimiento académico y otras variables, como quintil de ingreso.

En cuanto a los datos cualitativos, se realizaron dos focus group con los estudiantes becados, cuyo objetivo fue recabar información respecto a su participación en el CADE, los servicios que éste presta y la percepción del apoyo ofrecido para enfrentar la adaptación a las exigencias universitarias de manera exitosa. Los datos cualitativos recopilados fueron procesados mediante análisis de contenido. El procedimiento de análisis involucró distintas etapas, como el preanálisis, la definición de unidades de análisis y reglas, permitiendo la construcción de categorías, un aspecto central de esta técnica (CÁCERES, 2003). Las principales categorías construidas son:

a) adaptación a la vida universitaria;

b) percepción del programa CADE;

c) actores del programa: tutores y profesionales;

d) participación en el CADE y propuestas de mejora.

\section{PRINCIPALES RESULTADOS}

\section{Características sociodemográficas}

La distribución por sexo indica que el $68 \%$ de los estudiantes beneficiados con la BNA son mujeres, mientras que un $32 \%$ son hombres. En cuanto a la distribución por edad, el 53\% de los estudiantes tiene 19 años, seguido un 42\% con 18 años. La mayoría de los estudiantes provienen de zonas cercanas al campus principal de la universidad: un 87\% proviene de la Región del Bío-Bío, dentro 
de la cual la provincia de Concepción presenta el mayor número de estudiantes, seguida por las provincias de Arauco y Nuble. En cuanto al ingreso familiar, uno de cada dos estudiantes pertenece al quintil II, mientras que los quintiles I y II tienen una representación similar, como se observa en el Gráfico 1.

GRÁFICO 1 - Quintil de ingresos familiares de estudiantes beneficiarios

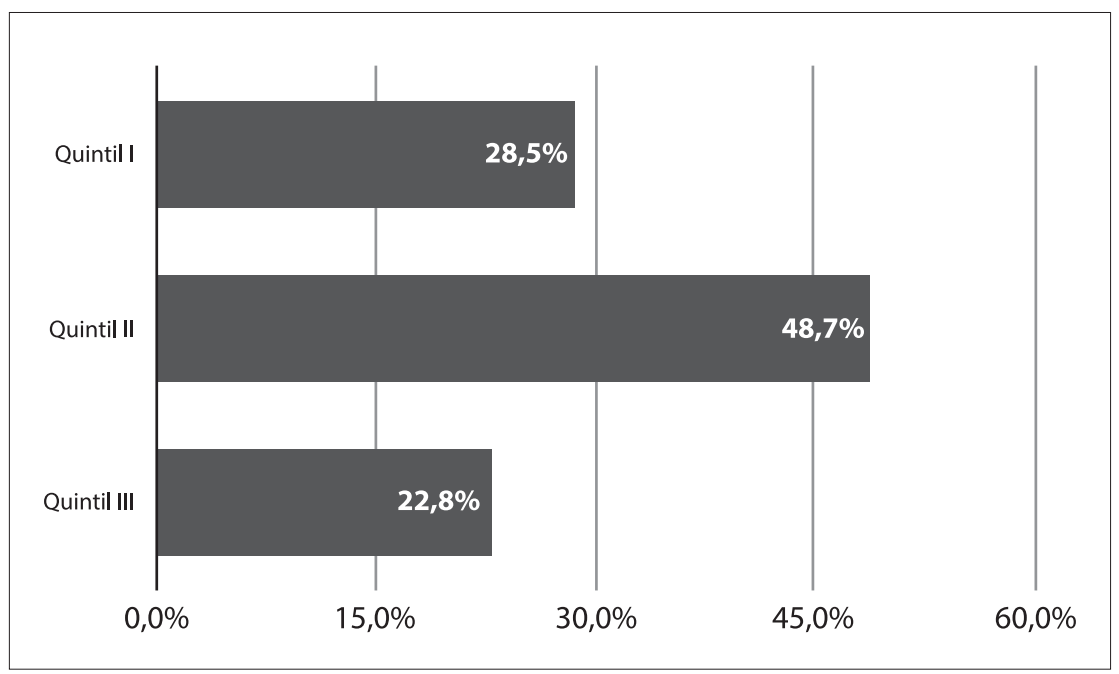

Fuente: Elaboración propia

Con relación al tipo de establecimiento donde los estudiantes realizaron su enseñanza secundaria, se destaca que un 54\% estudió en establecimientos municipales, seguido de un 46\% que estudió en establecimientos particulares subvencionados. En relación con la rama educacional de procedencia, el 89,3\% de los alumnos estudió en la Educación Científico-Humanista y un 10,7\% proviene de la Educación Técnico-Profesional. Esta distribución es coherente con los datos que indican que los estudiantes provenientes de la rama de educación Técnico-Profesional acceden en menor proporción a la universidad que los estudiantes de la educación Científico-Humanista (SEVILLA, 2011).

Respecto a las carreras cursadas por los alumnos becados, el 78\% se concentra en el área de Educación, seguido del área de Ingeniería y Administración, con un 17\%. Al realizar el cruce con la variable sexo, se observa que aquellas carreras vinculadas tradicionalmente al ámbito femenino concentran el mayor porcentaje de mujeres: Educación Diferencial y todas las Pedagogías, excepto Educación Física y Educación Musical. Esta distribución permite 
explicar el alto porcentaje de mujeres beneficiarias de la beca, ya que las carreras seleccionadas para participar en el programa son principalmente carreras de Pedagogía.

\section{ANTECEDENTES ACADÉMICOS}

El promedio de Notas de Enseñanza Media, según el tipo de establecimiento de egreso de los estudiantes, muestra que la media es muy similar en ambos casos (0.0019 punto levemente más alta para los estudiantes de establecimientos municipales). Del total de estudiantes, un $83 \%$ presenta notas entre 6.0 y 6.49 , en escala de 1.0 a 7.0, lo que se considera como una buena calificación.

Los puntajes de la Prueba de Selección Universitaria (PSU) muestran que los alumnos provenientes de establecimientos particulares subvencionados obtuvieron, en promedio, 33.6 puntos más que aquellos de establecimientos municipales. En la Tabla 2 se observa que los puntajes más bajos corresponden a alumnos pertenecientes a establecimientos municipales: uno de cada dos obtuvo puntajes entre 500 y 599 puntos; mientras que un 64\% de los estudiantes provenientes de establecimientos particulares subvencionados presentan puntajes entre 600 y 699 puntos.

TABLA 2 - Resultados en Prueba de Selección Universitaria por tipo de establecimiento escolar

\begin{tabular}{l|l|c|c|c|c}
\hline \multirow{2}{*}{$\begin{array}{l}\text { Tipo de } \\
\text { Establecimiento } \\
\text { Escolar }\end{array}$} & & \multicolumn{2}{|c|}{ Puntaje Prueba de Selección Universitaria } & \multirow{2}{*}{ Total } \\
\cline { 3 - 5 } & & $500-599$ & $600-699$ & $700-799$ & \\
\hline Municipal & $\mathrm{N}$ & 34 & 29 & 2 & 65 \\
& $\begin{array}{l}\text { \% Tipo de } \\
\text { Establecimiento }\end{array}$ & $52.3 \%$ & $44.6 \%$ & $3.1 \%$ & $100 \%$ \\
\multirow{2}{*}{ Part. Subvencionado } & $\mathrm{N}$ & 17 & 35 & 3 & 55 \\
& \% Tipo de & $30.9 \%$ & $63.6 \%$ & $5.5 \%$ & $100 \%$ \\
\multirow{2}{*}{ Total } & Establecimiento & 51 & 64 & 5 & 120 \\
& N & $42.5 \%$ & $53.3 \%$ & $4.2 \%$ & $100 \%$ \\
\hline
\end{tabular}

Fuente: Elaboración propia

Desagregado por sexo, el análisis de los promedios obtenidos en la PSU muestra que los hombres obtienen un promedio más alto en casi todas las pruebas, a excepción de matemáticas, en que las 
mujeres obtienen 15 puntos más en promedio por sobre los hombres. Sin embargo, al comparar el promedio de Notas de Enseñanza Media, se observa que las mujeres obtienen una media 15 puntos más alta por sobre los hombres.

\section{Rendimiento académico de los estudiantes beneficiarios de la BNA 2014}

El análisis de la situación académica de los estudiantes revela que, del total de beneficiados, un $86.6 \%$ continuó sus estudios en calidad de alumno regular de la universidad, es decir, se matriculó en el tercer semestre de su carrera; sólo el 13.4\% desertó (16 alumnos). Por tanto, se cumple la meta comprometida para el indicador de retención de primer año.

De los 104 estudiantes que continuaron sus estudios, 51\% provienen de establecimientos particulares subvencionados y $49 \%$, de establecimientos municipales. De aquellos que desertaron, un $25 \%$ proviene del ámbito municipal y un 75\% del sector particular subvencionado.

De acuerdo a los registros internos de la universidad, entre los principales antecedentes de la deserción, se encuentra la suspensión de estudios, ${ }^{8}$ seguida por baja por no inscripción, ${ }^{9}$ la renuncia a la universidad y la solicitud de cambio de carrera.

El análisis por sexo indica que igual número de hombres y mujeres abandona sus estudios (8), pero, como los beneficiarios hombres son menos numerosos, la deserción alcanza al 20.8\% en el subgrupo de hombres. Los motivos de deserción registrados por la universidad señalan que la mayoría de las mujeres deserta a través de la suspensión de estudios, mientras que sus pares masculinos presentan baja académica o no inscripción de ramos.

$\mathrm{El}$ análisis por quintiles de ingreso revela que los estudiantes pertenecientes al quintil II presentan mayor porcentaje de continuidad de estudios, con un $88.1 \%$. Al analizar las causas de deserción por quintil, se observa que la suspensión de estudios es más frecuente en el quintil III; la renuncia se observa en el quintil I; y la baja por no inscripción, en el quintil II. Por su parte, las carreras con mayor nivel de deserción corresponden a las Pedagogías en Inglés, Español, Ciencias Naturales y Matemática, seguido por Auditoria, Educación Parvularia y Biología Marina.

Respecto al rendimiento académico durante el primer año, del total de alumnos que continúan los estudios, un $62.5 \%$ presenta un promedio ponderado entre 5.0 a 5.9, como se observa en el Gráfico 2. El promedio ponderado para los estudiantes beneficiarios de la BNA es un 5.1, lo que implica superar la meta comprometida. 
GRÁFICO 2 - Promedio ponderado distribuido en tres categorías

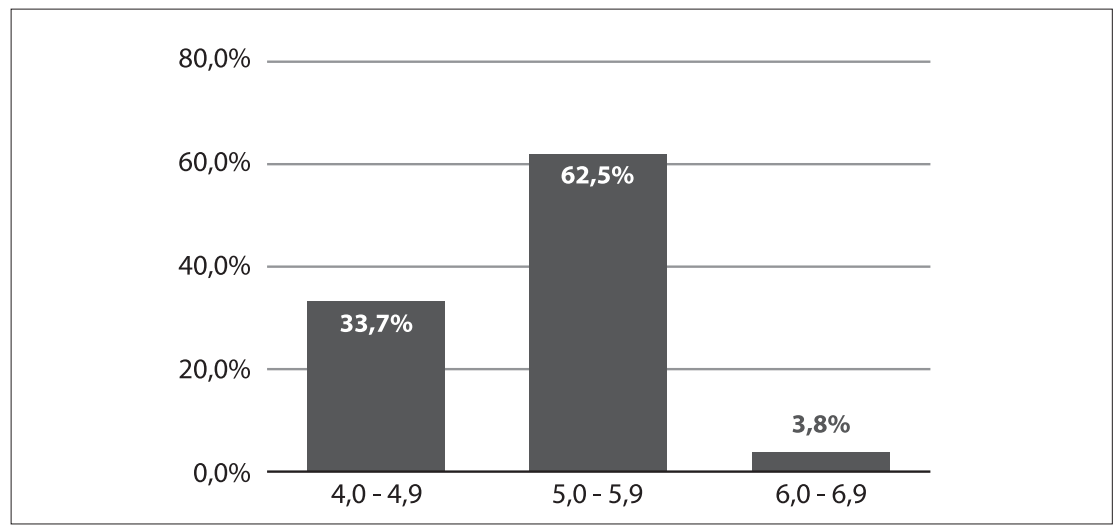

Fuente: Elaboración propia. ${ }^{10}$

En comparación con los resultados del primer semestre, se observa que existió una disminución de $4.9 \%$ entre aquellos alumnos con promedio bajo 4.5 , aumentando en un $1.8 \%$ el número de estudiantes en el tramo de nota entre 4.51 a 5.0. No obstante, el aumento más notorio se observa en el rango 5.0 a 5.9, en que se encuentra un $16.8 \%$ más de alumnos que en el primer semestre.

Un análisis más detallado de los resultados académicos revela que un 49\% de los estudiantes con BNA aprobó todas sus asignaturas, con una media de 10 asignaturas aprobadas. Este porcentaje implica el cumplimiento del indicador que señalaba como meta el logro del $30 \%$ de los beneficiarios con todos sus créditos aprobados.

Del total de becados que continúa estudios, pero que reprobó alguna asignatura, se observan los detalles en el Gráfico 3.

GRÁFICO 3 - Número de asignaturas reprobadas en estudiantes que continúan estudios

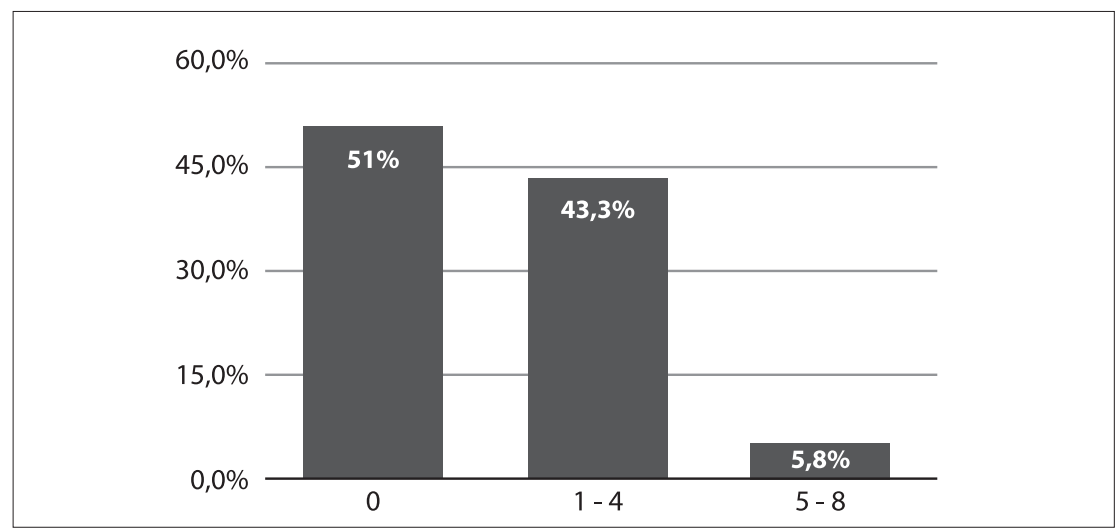

Fuente: Elaboración propia 
Las características sociodemográficas y académicas de los estudiantes tienen un impacto estadísticamente significativo, aunque limitado, en sus resultados académicos, como se concluye de la realización de la prueba estadística de Spearman, que permitió establecer correlaciones entre las variables ya descritas.

Los resultados indican que existe una correlación directa entre el quintil de ingresos y el número de asignaturas aprobadas, así como también una correlación fuerte e inversamente proporcional entre el quintil y el número de asignaturas reprobadas, es decir, a más bajo quintil de ingresos del alumno, mayor número de asignaturas reprobadas.

El promedio ponderado de notas ${ }^{11}$ es más alto entre aquellos del quintil III (5.29), seguido de cerca por aquellos alumnos del quintil I (5.19), mientras que, para aquellos alumnos del quintil II, el promedio ponderado está por debajo del 5.0. La mayor cantidad de asignaturas aprobadas corresponde a estudiantes pertenecientes al tercer quintil de ingreso con una diferencia por tramo de 0.9 punto. $\mathrm{Al}$ mismo tiempo, la mayor cantidad de asignaturas reprobadas se presenta en alumnos pertenecientes al quintil I.

Se observa una correlación positiva y estadísticamente significativa entre el puntaje obtenido en la prueba de hábitos de estudio y el promedio ponderado de notas y, separadamente, con los resultados de aprobación de asignaturas. En otras palabras, a más bajo resultado obtenido en la prueba de hábitos de estudio, menor promedio ponderado de notas y menor aprobación de asignaturas. Los puntajes de la prueba de comunicación y de la prueba de capacidad para la toma de decisiones también se correlacionan significativamente con el número de asignaturas aprobadas. Los resultados obtenidos en las pruebas de hábitos de estudio, de comunicación y de toma de decisiones constituyen variables predictivas de los resultados académicos alcanzados durante el primer año de universidad.

Para comparar los logros académicos según establecimiento de procedencia, municipal o particular subvencionado, se aplicó la Prueba $\mathrm{T}$ a las variables promedio ponderado de notas, promedio curricular, créditos aprobados y asignaturas aprobadas, concluyendo que no existen diferencias significativas $(\mathrm{p}<0.05)$. No hay evidencia suficiente para afirmar que los resultados académicos de los estudiantes sean distintos por tipo de establecimiento de enseñanza secundaria.

El cruce de los resultados académicos según quintil de pertenencia indica que sólo existe homocedasticidad (igualdad de varianzas) para la variable "promedio ponderado obtenido segundo semestre", por lo que, para analizarla, se utilizó ANOVA. Para las otras variables, se utilizó la prueba Welch. 
A un nivel de confianza del 95\%, no se encuentra evidencia suficiente para afirmar que existen diferencias entre el promedio ponderado de notas obtenido en el segundo semestre según quintil de los estudiantes becados. Este dato ratifica los resultados de otras pruebas.

El análisis a partir de la prueba Welch indica que, a un nivel de significación de 0.05 , o sea, un nivel de confianza del $95 \%$, sólo se observan diferencias para el porcentaje de asignaturas aprobadas. Es decir, al menos uno de los quintiles tiene un porcentaje de asignaturas aprobadas significativamente diferente. Con un nivel de significación de 0.01 (y nivel confianza del 99\%), es posible afirmar que existe alguna o algunas diferencias significativas entre los quintiles en la variable porcentaje de créditos aprobados. Por otro lado, el análisis no encuentra diferencias significativas en el promedio de notas según quintil de ingresos.

Una comparación similar se aplicó a los promedios de créditos aprobados por los estudiantes. Para analizar esta variable en detalle y determinar si existen diferencias entre los grupos por quintil de ingreso, se utilizó la prueba de comparaciones múltiples GamesHowell. El análisis arroja una diferencia estadísticamente significativa, $\mathrm{p}<0.05$, aunque mínima entre los promedios de créditos aprobados de los quintiles I y III, pero no entre los quintiles I y II o II y III.

En resumen, si bien se puede afirmar que el nivel socioeconómico de los estudiantes tiene un impacto en su rendimiento académico, este impacto es limitado por sí solo. No obstante, cabe mencionar que en este análisis sólo se incluyen los estudiantes beneficiados, todos de los tres quintiles más bajos, de modo que no es posible establecer conclusiones respecto a las diferencias entre el desempeño de estos estudiantes y aquellos pertenecientes a grupos socioeconómicos altos.

\section{PERCEPCIONES DE LOS ESTUDIANTES RESPECTO AL CÍRCULO-CADE}

Al finalizar el año académico, se aplicó una encuesta en línea, anónima y voluntaria a los estudiantes del Círculo-CADE y se realizaron dos focus group. Ambas técnicas de recolección de datos tenían por objetivo identificar las percepciones de los estudiantes respecto a su participación en el programa.

El focus group permitió analizar el proceso de adaptación a la vida universitaria desde la perspectiva de los estudiantes. Uno de los aspectos abordados en sus relatos se refiere a las diferencias existentes entre la enseñanza secundaria y la universidad. En este sentido, se mencionan falencias en la formación escolar y debilidad o ausencia de estrategias de aprendizaje. La demanda por compatibilizar las exigencias de distintas asignaturas implicó para muchos un cambio significativo: 
En el liceo uno se acostumbra a las buenas notas y pasar, por ejemplo, de un 6 a un 4 igual [...] es mucho el cambio [...] en el liceo yo no estudiaba tanto pero igual me iba bien, pero aquí dije, me decían "la universidad es distinto, tienes que estudiar más". (Participante 1).

Uno no le tomaba el peso, pero, cuando uno lo vive, sí, es realmente una etapa diferente. Para mí al menos, en cierta forma, ha sido liberador estar acá, porque como que no hay tantas reglas y esto y esto otro, pero siempre viendo por mí y que ojalá me vaya bien en todo, pero igual me frustro demasiado cuando son bajas las notas. (Participante 4).

En este contexto, existe un grupo mayoritario que considera la pertenencia al programa Círculo-CADE como un factor que potenció su desarrollo académico. Los resultados de la encuesta indican que un $47 \%$ volvería a participar de un programa como el Círculo-CADE con toda seguridad.

[...] yo lo veo más como la oportunidad de aprovechar un beneficio, entonces uno para un beneficio igual tiene que cumplir ciertos requisitos. Yo creo que los requisitos son los mínimos para los beneficios que se dan. (Participante 9).

Respecto a las actividades realizadas por CADE, los resultados indican que los talleres obtienen la mejor evaluación, con un $72 \%$ de percepciones positivas. El elemento más relevante para los becados es la actitud de los expositores, considerada amable y accesible; mientras que la claridad de la información entregada fue importante para el 19\% de los encuestados. A pesar de estos resultados, un 28\% considera que los talleres no constituyen una actividad de interés, situación que es explicada, en parte, por la didáctica utilizada. En opinión de un estudiante, la asistencia a los talleres:

Me ayudó bastante. Por un lado, las charlas a las que asistí me ayudaron en el ámbito académico, me ayudaron a desarrollarme mejor en algunos aspectos de mi vida como con el taller de comunicación y concentración y que me ayudaron mucho en mis notas. (Participantes 5).

La percepción acerca de las atenciones de los profesionales del equipo CADE muestra que un 91\% de los encuestados las califican como rápidas y eficientes, ya que brindan soluciones a sus inquietudes, mientras que un $84 \%$ considera fácil conseguir una cita. Un ejemplo de esta opinión es la siguiente:

Yo ahora fui armándome los hábitos de estudio, con el psicopedagogo. Primero fue la psicóloga académica del CADE que me ayudó a organizarme, con una charla para organizarse. Y ya ahí organicé mi tiempo con ella y ahora con el psicopedagogo que me ha estado ayudando con los hábitos de estudio y las técnicas de estudio. (Participante 9). 
El relato de los estudiantes permite concluir que algunos de ellos requieren mayor apoyo psicológico y psicopedagógico para lograr adaptarse a las exigencias académicas, fenómeno que debiera considerarse en futuros proyectos.

Llega un momento en que [...], hace un mes atrás, en que uno colapsa totalmente, uno estudia mucho, y nos hacen test casi todas las semanas, yo tengo 5 y 6 , entonces llegó el día del certamen y yo contesté la mitad de la prueba porque me quedé en blanco, y siendo que yo sabía todo. Entonces como que esa parte es frustrante porque igual uno tiene un rojo y después [...] entonces con las otras materias que igual a uno le cuestan más [...] no me va tan bien como yo espero. (Participante 3).

Los tutores CADE también tienen un rol fundamental en el desarrollo del programa. La encuesta indica que el conocimiento de las funciones asociadas a la labor del tutor tiene una alta valoración en opinión del 93\% de los encuestados. Asimismo, el 88\% de los encuestados considera que su tutor conocía dónde y a quién derivar a los estudiantes que requerían otro tipo de apoyo. Estos datos indican que la labor del tutor no sólo es relevante para la inducción a la vida universitaria, sino también para apoyar el trabajo realizado por el equipo profesional del programa, como lo demuestran los siguientes estudiantes.

Estoy muy contenta con mi tutora porque, a pesar de que estaba ocupada, se preocupaba de que nosotras contáramos con su apoyo, y también los profesionales del CADE siempre estuvieron ahí cuando los necesitamos. El CADE fue una ayuda y apoyo para mis estudios. (Participante 3).

Por ejemplo, a mi tutor [...] siempre nos está avisando, nos estamos juntando semanalmente, nos dice "chiquillos, si tienen duda en alguna materia, nos juntamos a estudiar en el auditorio del CADE". Incluso tenemos un grupo en Facebook, con tutorial [risas] y entonces estamos siempre en contacto. (Participante 1).

Respecto a la organización del trabajo del tutor, el 91\% de los estudiantes indican que cuentan con un plan de trabajo, la disposición de los tutores para ayudar y claridad en sus funciones. Durante las sesiones de tutoría, un 84\% de los encuestados encontró una solución a sus inquietudes. En consecuencia, el tutor juega un rol importante de acompañamiento y referencia para la adaptación de los estudiantes a la vida universitaria.

Como que conocí más la universidad, gracias a ellos [...] compartí con más gente, informarnos sobre la carrera y todo eso. (Participante 5).

Respecto a la necesidad de realizar cambios para futuras ejecuciones del programa, un 37\% de los encuestados indica que no mejoraría ningún aspecto. En cambio, otros estudiantes identifican un ámbito que limita el desarrollo satisfactorio del programa: los 
horarios. En particular, mencionaron la dificultad de compatibilizar los horarios de los estudiantes y la planificación de las charlas y talleres ofrecidos por CADE. Por ello, la petición de los estudiantes es flexibilizar la planificación horaria del CADE:

[...] cuando empecé a ir el segundo mes, a mí me sirvieron mucho los talleres y yo me arrepentí de no ir el primer mes, pero fue porque no me calzaba ningún horario. (Participante 3).

Adicionalmente, algunos encuestados plantean la necesidad de aumentar las horas de atención psicológica y psicopedagógica, así como de incorporar espacios de estudio individual, aumentar la disponibilidad de horas de trabajo de los tutores, aumentar la beca para fotocopias y mejorar la didáctica de los talleres, todas con un $3 \%$.

\section{DISCUSIÓN Y CONCLUSIONES}

El programa Beca de Nivelación Académica fue una de las primeras iniciativas del Ministerio de Educación para mejorar los indicadores de deserción y retención. Ambos fenómenos se vinculan al problema de la equidad de la educación superior caracterizado por un acceso masificado de estudiantes de origen vulnerable, pero una limitada retención y, por ende, baja titulación.

Este programa marca un punto de inflexión en el curso de las políticas públicas, revelando un cambio en la concepción del éxito y fracaso académico en la universidad, desde el fracaso como responsabilidad individual, como lo plantea Donoso-Díaz, Donoso y Arias (2010), al fracaso como una responsabilidad colectiva, de los estudiantes, las carreras y de la universidad como institución. Igualmente, en lugar de establecer más políticas de corte económico, se impulsa un programa de apoyo académico y de inducción a la vida universitaria, reconociendo que la deserción involucra otros factores además de los económicos.

El Ministerio de Educación estableció las bases del programa definiendo indicadores y metas a lograr. No obstante, son las universidades, mediante la elaboración de un proyecto, quienes deben proponer estrategias y acciones destinadas a lograrlos; por tanto, pueden existir diferencias entre instituciones en los procedimientos para alcanzar objetivos comunes.

La Universidad de Concepción definió una serie de acciones destinadas a apoyar a los estudiantes beneficiarios del programa. Entre estas acciones se destacan:

a) la realización de charlas y talleres que buscan desarrollar competencias básicas; 
b) la incorporación de tutores para apoyar la inducción a la vida universitaria y monitorear el rendimiento académico de los estudiantes; c) el apoyo de una red de profesionales, como asistente social, psicólogo y psicopedagogo.

Los resultados de la intervención revelan que se ha logrado una retención del $86.6 \%$, alcanzando la meta propuesta por el programa. Asimismo, se observó un aumento en los promedios obtenidos por los estudiantes en el transcurso del año, dando cuenta de una adaptación gradual pero consistente a las exigencias académicas de la universidad. Otro programa BNA implementado por una universidad también ha revelado el aumento de notas y mejoramiento en el nivel de aprobación de los beneficiarios (MICIN et al., 2015).

En el proceso de adaptación concurren elementos propios de la vida personal y del origen sociofamiliar y cultural de cada estudiante. Tal como fue identificado por Canales y De Los Ríos (2009) y Leyton, Vásquez y Fuenzalida (2012), los participantes del focus group perciben deficiencias en su formación escolar que son entendidos como obstáculos para finalizar su primer año y que conllevan sentimientos de incertidumbre y frustración. En este contexto, los beneficiarios valoran el programa CADE gracias a las distintas acciones destinadas a enfrentar exitosamente los desafíos de su nuevo estatus de estudiante universitario. Por tanto, el programa funciona como un facilitador de la adaptación de los estudiantes a las exigencias académicas de la universidad. En atención a estos antecedentes, se hace necesario visibilizar la labor del CADE, socializando sus actividades para posesionarlo como un referente, no sólo para los beneficiarios de la BNA, sino también para todos los estudiantes de la universidad, jefes de carreras y departamentos.

El análisis de los datos indica que existe una correlación entre el quintil de ingresos y el número de asignaturas aprobadas, así como también entre el quintil de ingresos y el número de asignaturas reprobadas. No obstante, el quintil de ingresos no permite explicar por sí solo el rendimiento académico. Estos datos muestran, por un lado, la importancia de realizar nuevas investigaciones que analicen las variables que determinan el rendimiento académico de estudiantes provenientes de familias de bajos ingresos. Por otro lado, revelan la importancia de fortalecer programas como la BNA para apoyar a estos estudiantes, de modo que sus logros en la universidad no estén determinados por la posición social de sus familias.

La prueba de hábitos de estudios demostró ser una variable predictiva del rendimiento académico en este grupo de estudiantes. Futuros análisis deberían determinar si estos resultados son válidos para la población estudiantil. De verificarse, la prueba de hábitos 
de estudio puede constituirse en una herramienta de intervención destinada a identificar a jóvenes que potencialmente puedan presentar problemas de rendimiento académico. En caso de bajo puntaje en la prueba, los estudiantes debieran asistir a los talleres orientados a desarrollar hábitos de estudio. De igual forma, sería necesario priorizar su participación en las tutorías y buscar mecanismos para asegurar una atención integral por parte del equipo CADE.

Los "nuevos estudiantes" que acogen actualmente la educación superior poseen perfiles socioeconómicos y académicos distintos a lo que se observaba cuando este nivel educativo estaba destinado a una élite. El acceso masificado de estudiantes de origen vulnerable a las universidades chilenas demanda que estas instituciones desarrollen mecanismos de diversa índole que garanticen equidad en los resultados de retención y titulación. En este sentido, las instituciones, además de crear o fortalecer instancias de inducción a la vida universitaria y apoyo académico, pueden desarrollar estrategias transversales para favorecer la equidad en educación superior.

Recientemente, el Ministerio de Educación ha promovido la realización de Convenios de Desempeño ${ }^{12}$ para producir cambios relevantes en distintos ámbitos, como la armonización curricular y la formación de profesores. En la Universidad de Concepción, el Convenio de Desempeño "Profesores UdeC: Protagonistas del cambio en la sociedad del conocimiento" (2013-2015) generó cambios importantes en la estructura curricular de las catorce carreras de formación inicial docente. Desde el punto de vista de la equidad, se destaca la incorporación en la malla curricular de un curso de Español Académico. Esta decisión se basó en un diagnóstico que reveló la necesidad de fortalecer competencias comunicativas en estudiantes de primer año. El curso busca entregar herramientas básicas a los estudiantes para cursar exitosamente sus estudios universitarios.

Una estrategia desarrollada por las universidades ha sido focalizar las acciones en carreras que presentan alta deserción. Un ejemplo de aquello es el programa Tutores de la Universidad de Bío-Bío, que buscó disminuir la tasa de deserción de carreras de ciencias básicas, particularmente durante los primeros años. El programa realizó un conjunto de acciones lideradas por estudiantes de cursos superiores - llamados tutores- quienes cumplían un rol de acompañamiento y conserjería a sus compañeros de primer año, de modo de facilitar su integración en la educación superior y favorecer su éxito académico. La experiencia de este programa demostró que el trabajo de apoyo académico entre estudiantes de primer año y cursos superiores puede tener resultados positivos en la disminución 
de la deserción y el mejoramiento del rendimiento académico (ver Universidad del Bío-Bío, s/f).

La gestión de información también puede constituir una herramienta para facilitar la identificación de estudiantes en riesgo de deserción. Distintas universidades chilenas han comenzado a implementar sistemas de alerta que reúnen variables académicas de todos estudiantes en una plataforma informática, permitiendo realizar un monitoreo del rendimiento académico y alertar a los docentes y autoridades de las carreras acerca de los estudiantes que se encuentren en riesgo de reprobar una o más asignaturas y/o de no cumplir con los requisitos académicos para permanecer en la universidad. Mecanismos de esta naturaleza permiten a los docentes apoyar a sus estudiantes y utilizar efectivamente la información disponible en los registros institucionales.

\section{REFERENCIAS}

ALVARADO, V. et al. Diversidad y equidad: programas de acción afirmativa en la educación superior chilena. En: GONZÁLEZ, L. E. (ed.). Diagnóstico y diseño de intervenciones en equidad universitaria. Santiago de Chile: Centro Interuniversitario de Desarrollo, p. 193-229, 2010.

BEAUD, S. 80\% au bac... et après. Les enfants de la démocratisation scolaire. París: La Découverte, 2002. BERNASCONI, A.; ROJAS, F. Informe de Educación Superior 1980-2003. Santiago de Chile: Universitaria, 2004.

BRUNNER, J. J. Educación superior en Chile: instituciones, mercados y políticas gubernamentales (1967-2007). Santiago de Chile: Ediciones Diego Portales, 2009.

CÁCERES, P. Análisis cualitativo de contenido: una alternativa metodológica alcanzable. Psicoperspectivas, Valparaíso, v. 2, n. 1, p. 53-82, 2003.

CANALES, A.; DE LOS RÍOS, D. Retención de estudiantes universitarios vulnerables. Calidad en la Educación, Santiago de Chile, n. 30, p. 50-83, jul. 2009.

CANALES, A.; DE LOS RÍOS, D. Factores explicativos de la deserción universitaria. Calidad en la Educación, Santiago de Chile, n. 26, p. 173-201, jul. 2007.

CASTILLO, J.; CABEZAS, G. Caracterización de jóvenes primera generación en educación superior: nuevas trayectorias hacia la equidad educativa. Calidad en la Educación, Santiago de Chile, n. 32, p. 44-76, jul. 2010.

CENTRO DE MICRODATOS. Estudio sobre causas de la deserción universitaria. Informe final. Santiago de Chile: Departamento de Economía, Universidad de Chile, 2008.

CENTRO DE POLÍTICAS COMPARADAS DE EDUCACIÓN - CPCE. Primera generación en la universidad. Informe preliminar. Santiago de Chile: Universidad Diego Portales, 2009.

CHEN, X.; CARROLL, C. First-Generation Students in Postsecondary Education: A Look at Their College Transcripts Postsecondary Education. Descriptive Analysis Report. Project Officer. Washington, DC: National Center for Education Statistics, 2005.

CHILE. Ministerio de Desarrollo Social. Encuesta de Caracterización Socioeconómica Nacional - CASEN. 2016. Disponible en: <http://observatorio.ministeriodesarrollosocial.gob.cl/ casen/casen_def_ingresos.php>. Consultado el: 15 en. 2016 
CHILE. Ministerio de Educación. ¿Qué es un Convenio de Desempeño? 2015. Disponible en: <http://www.mecesup.mineduc.cl/index2.php?id_portal=59\&id_seccion=3605\&id_ contenido $=14986>$. Consultado el: 27 enero, 2016

CHILE. Ministerio de Educación. Términos de referencia. Convocatoria: Programa de Nivelación Académica para estudiantes de Primer Año de Educación Superior - año 2014. 2014. Disponible en: <http://www.mecesup.cl/index2.php?id_seccion=4212\&id_ portal=59\&id_contenido $=30225>$. Consultado el: 23 oct. 2016.

CONSEJO NACIONALDE EDUCACIÓN - CNED. Educación Superior: conceptos básicos. 2015. Disponible en: <http://www.cned.cl/public/secciones/SeccionEducacionSuperior/ conceptos_basicos.aspx>. Consultado el: 25 enero 2016.

CONTRERAS, M.; CORBALÁN, F.; REDONDO, J. Cuando la suerte está echada: estudio cuantitativo de los factores asociados al rendimiento de la PSU. Santiago de Chile: Facultad de Ciencias Sociales, Universidad de Chile, 2007.

DONOSO-DÍAZ, S.; CÁNCINO, V. Caracterización socioeconómica de los estudiantes de educación superior. Calidad en la Educación, Santiago de Chile, n. 26, p. 205-244, enero 2007.

DONOSO-DÍAZ, S.; DONOSO, G.; ARIAS, O. Iniciativas de retención de estudiantes en educación superior. Calidad en la Educación, Santiago de Chile, n. 33, p. 15-61, dic. 2010.

ESPINOZA, O. Equidad en el Sistema de Educación Superior en Chile desde la Perspectiva de los Resultados. Propuesta Educativa, Buenos Aires, n. 43, p. 46-64, jun. 2015.

ESPINOZA, O.; GONZÁLEZ, L.; LATORRE, C. Un modelo de equidad para la educación superior: análisis de su aplicación al caso chileno. Revista de la Educación Superior, México, D.F., v. 38, n. 150, p. 97-112, abr./jun. 2009.

GROLEAU, A. et al. Les possibilités et les limites d'une importation conceptuelle. Les étudiants de première génération au Canada. Education et Sociétés, París, n. 26, p. 107-122, 2010.

KOLJATIC, M.; SILVA, M. Algunas reflexiones a siete años de la implementación de la PSU. Estudios Públicos, Santiago de Chile, n. 120, p. 125-146, 2010.

LETELIER, M. et al. Gestión de la calidad en Educación Superior. En: GONZÁLEZ, L. E. (ed.). Diagnóstico y diseño de intervenciones en equidad universitaria. Santiago de Chile: Centro Interuniversitario de Desarrollo, 2010. p. 91-107.

LEYTON, D.; VÁSQUEZ, A.; FUENZALIDA, V. La experiencia de estudiantes de contextos vulnerables en diferentes instituciones de Educación Superior Universitaria (IESU): resultados de investigación. Calidad en la Educación, Santiago de Chile, n. 37, p. 61- 97, dic. 2012.

MANZI, J. El acceso segmentado a la educación superior en Chile. En: DÍAZ-ROMERO, P. (Comp.). Caminos para la inclusión en la educación superior. Santiago de Chile: Fundación Equitas, 2006. p. 187-204.

MICIN, S. et al. Beca de Nivelación Académica: la experiencia de una política pública aplica en una universidad chilena. Calidad en la Educación, Santiago de Chile, n. 42, p. 189-208, jul. 2015.

ORGANIZACIÓN PARA LA COOPERACIÓN Y EL DESARROLLO ECONÓMICO - OCDE; BANCO INTERNACIONAL PARA LA RECONSTRUCCIÓN Y EL DESARROLLO - BANCO MUNDIAL. La Educación Superior en Chile - Revisión de Políticas Nacionales de Educación. Traducción de Ministerio de Educación de Chile. Santiago de Chile: Ministerio de Educación de Chile, 2009.

PEARSON. Evaluación de la PSU Chile. Informe final. Resumen ejecutivo. 2013. Disponible en: <http://portales.mineduc.cl/usuarios/mineduc/doc/201301311058200.ChilePSUResumen_Ejecutivo.pdf $>$. Consultado el: 05 oct. 2015 
PROGRAMA DE NACIONES UNIDAS PARA EL DESARROLLO - PNUD. Expansión de la educación superior en Chile: hacia un nuevo enfoque de la equidad y la calidad. Temas de Desarrollo Humano Sustentable, Santiago de Chile, n. 10, p. 1-95, 2005.

ROMÁN, C. Más programas propedéuticos en Chile: el discurso de los estudiantes en la Universidad Católica Silva Henríquez. Estudios Pedagógicos, Valdivia, v. 39, n. 2, p. 263-278, 2013.

SERVICIO DE INFORMACIÓN DE EDUCACIÓN SUPERIOR - SIES. Panorama de la Educación Superior en Chile 2014. Santiago de Chile: División de Educación Superior, Ministerio de Educación, 2014.

SEVILLA, P. Diagnóstico a la Educación Técnico-Profesional. Santiago de Chile: Ministerio de Educación, 2011.

UNIVERSIDAD DEL BÍO-BÍO. Programa Tutores. Disponible en: < http://convenio. ubiobio.cl/capsulas/tutores.html/>. Consultado el: 27 enero, 2016.

UNIVERSIDAD DE CONCEPCIÓN. Reglamento General de Docencia de Pregrado. Decreto UdeC n $n^{\circ}$ 2008-116. Aprueba la modificación orgánica al Reglamento de Docencia de Pregrado, que se denominará en adelante Reglamento General de Docencia de Pregrado. Concepción, 2008.

URZÚA, S. Rentabilidad de la educación superior en Chile: revisión de las bases de 30 años de políticas públicas. Estudios Públicos, Santiago de Chile, n. 125, p.1-52, 2012.

WARBURTON, E. et al. Bridging the Gap Academic Preparation and Postsecondary Success of FirstGeneration Students. Washington, D.C.: National Center for Education Statistics, 2001.

ZAPATA, G. (Coord.); TEJEDA, I.; ROJAS, Á. Educación Superior en Chile. Informe Nacional. En: JOAQUÍN, J.; HURTADO, R (ed.). Educación Superior en Ibero América: Informe 2011. Santiago de Chile: Centro Interuniversitario de Desarrollo, 2011.

\section{NOTAS}

${ }^{1}$ En Chile existen dos modalidades educativas: Científico-Humanista y Técnico-Profesional. De acuerdo a Sevilla (2011), la rama Técnico-Profesional representa un 45\% de la matrícula de educación media y agrupa principalmente a los tres quintiles de más bajos ingresos.

${ }^{2}$ El sistema de educación superior chileno se compone por tres tipos de instituciones: Universidades, Institutos Profesionales y Centros de Formación Técnica. Las Universidades otorgan toda clase de títulos y grados académicos. Los Institutos sólo entregan títulos profesionales y diplomas de formación técnica. Los Centros de Formación Técnica entregan títulos técnicos (CONSEJO NACIONAL DE EDUCACIÓN, 2015).

${ }^{3}$ Para Brunner (2009), la estratificación escalonada se explica por una oferta de vacantes en las universidades que sobrepasa anualmente la demanda, por lo cual un grupo de universidades poco selectivas no puede rechazar postulantes.

${ }^{4}$ Las carreras focalizadas, como las denomina el MINEDUC, fueron escogidas en base a dos criterios: primero, carreras con menos del $70 \%$ de tasa de retención, y segundo, todas las carreras de pedagogía ofrecidas por la Universidad de Concepción. Este último criterio tuvo la finalidad de apoyar el logro de los indicadores de otro proyecto de la Universidad y el MINEDUC destinado a fortalecer la formación inicial docente. (Ver <http://docencia. udec.cl/unidd/index.php?option $=$ com_content\&view $=$ article\&id $=77 \&$ Itemid $=30>$ ).

${ }^{5}$ Definición de Quintil de Ingreso: la Encuesta de Caracterización Socioeconómica Nacional indica que el Quintil del Ingreso autónomo per cápita del hogar nacional corresponde a la quinta parte o $20 \%$ de los hogares nacionales ordenados en forma ascendente de acuerdo al ingreso autónomo 
per cápita del hogar, en que el primer quintil (quintil I) representa el 20\% más pobre de los hogares del país, y el quinto quintil (quintil V), el 20\% más rico de estos hogares. (CHILE, 2016).

${ }^{6}$ Los objetivos y acciones se encuentran en el Formulario de Postulación. Convocatoria: Programa de Nivelación Académica para Estudiantes de Primer Año de Educación Superior. Beca de Nivelación Académica - año 2014.

${ }^{7}$ Agronomía, Auditoría, Biología, Biología Marina, Ingeniería en Biotecnología Vegetal, Ingeniería en Biotecnología y Acuicultura, Ingeniería en Conservación de Recursos Naturales, Ingeniería Forestal, Educación General Básica, Educación Parvularia, Educación Diferencial, Pedagogía en Artes Plásticas, Pedagogía en Ciencias Naturales y Biología, Pedagogía en Ciencias Naturales y Física, Pedagogía en Ciencias Naturales y Química, Pedagogía en Educación Física, Pedagogía en Educación Musical, Pedagogía en Español, Pedagogía en Filosofía, Pedagogía en Historia y Geografía, Pedagogía en Inglés y Pedagogía en Matemática y Computación.

${ }^{8}$ Suspensión de estudios es definida por el Reglamento General de Docencia de Pregrado, Decreto UdeC 2008-116, como sigue: "Los alumnos de pregrado podrán solicitar, al Vicedecano, suspender estudios hasta cuatro semanas antes del término del periodo lectivo respecto. La suspensión de estudios deja sin efecto la inscripción de asignaturas e interrumpe la calidad de alumno de pregrado de la Universidad de Concepción”.

${ }^{9}$ Baja por no inscripción es definida por el Reglamento de Docencia como sigue: "Alumnos que estando habilitados para inscribir asignaturas no lo hagan dentro del periodo correspondiente perderán el derecho a continuar estudios como también la calidad de alumno de pregrado de la Universidad de Concepción, a menos que en el periodo lectivo anterior hayan inscrito una o más asignaturas anuales".

${ }^{10}$ La nota 7.0 no fue incluida para que los intervalos fueran idénticos. Esto no ocasiona problemas, puesto que ningún estudiante obtuvo nota 7.0.

${ }^{11}$ Promedio ponderado de notas, según Reglamento General de Docencia de Pregrado, se refiere a "promedio de las notas finales iguales o superiores a 4.0, ponderadas por el correspondiente número de créditos".

${ }^{12}$ Un Convenio de Desempeño es "un contrato entre el Estado y las Instituciones de Educación Superior por medio del cual éstas comprometen desempeños notables que impliquen un significativo mejoramiento institucional, que por sí sola no habrían podido lograr" (CHILE, 2015).

Submetido: $19 / 02 / 2016$

Aprovado: 23/05/2016

Contato:

Valentina Soto Hernández Avenida Eix Central. Edificio B. Cerdanyola del Vallès. Barcelona España, 08193

Dirección de Docencia. Edmundo Larenas, 64A

Universidad de Concepción Concepción, Chile 